\title{
Evaluation of BACTEC MGIT 960 system for recovery of Mycobacterium tuberculosis complex in Pakistan
}

\author{
Luqman Satti, * Aamer Ikram, Shahid Abbasi, Tariq Butt, Nasarullah Malik and Irfan Ali Mirza \\ Department of Microbiology, Armed Forces Institute of Pathology (AFIP), Rawalpindi, Pakistan. \\ E-mail: luqmansatti@hotmail.com
}

Received 4 December 2009; received in revised form 26 February 2010; accepted 4 March 2010

\begin{abstract}
We evaluated the performance of MGIT 960 system in terms of recover rate, detection time of mycobacteria and contamination rate from various human clinical specimens and compared it with already in use BACTEC 460 TB system and conventional LJ medium. This is the first reported study on MGIT 960 and its comparison with BACTEC 460 system in Pakistan. A total of 260 different clinical specimens received for the culture of mycobacteria were dealt during the six months study period. All the specimens were digested and decontaminated according to the standard N-acetyl-Lcysteine $\mathrm{NaOH}$ method. All the processed specimens were inoculated on both the liquid systems and solid medium and incubated for six weeks and eight weeks consecutively. A total of 44 mycobacterial isolates (Mycobacterium tuberculosis, $n=43$; Mycobacteria other than tuberculosis, $n=1$ ) were recovered from 260 clinical specimens. The recovery rate of $M$. tuberculosis complex was $97.6 \%$ on BACTEC MGIT 960 system and $93.0 \%$ on BACTEC 460 system and $83.7 \%$ on LJ medium. The mean detection time of mycobacteria on BACTEC MGIT 960 system was 11.2 days in smear positive cases, 14.2 days in smear negative cases and 14.8 days in smear positive cases on BACTEC 460 system. Contamination rates were $9.6 \%$ and $5.6 \%$ and $3.4 \%$ for BACTEC MGIT 960, BACTEC 460 system and LJ medium respectively. The non-radiometric, fully automated BACTEC MGIT 960 system has better diagnostic ability as compared with radiometric, semi-automated BACTEC 460 system and LJ medium, so it can be used as a reliable alternative in over burden laboratories.
\end{abstract}

Keywords: BACTEC, tuberculosis, Mycobacterium tuberculosis complex.

\section{INTRODUCTION}

Pakistan ranks sixth among the high TB burden countries with incidence and prevalence rate of $181 / 100,000$ and 359/100,000 population (Hasan et al., 2006). The growing number of tuberculosis cases specially in underdeveloped countries has made it necessary to develop new tools for rapid detection and identification of mycobacteria from various clinical samples (Hanna et al., 1999). In resource poor countries, direct AFB microscopy and Lowenstein Jensen (LJ) media are still main modalities used for the diagnosis/screening of tuberculosis, but these methods have low sensitivity which also depends upon the number of tubercle bacilli present in the specimen (Farnia et al., 2002). In some areas, patients are often treated on the presumptive diagnosis due to lack of diagnostic facilities. The broth based BACTEC 460 system, introduced in 1980 , has considerably improved the detection time of mycobacteria and it was a milestone in the advancement in mycobacteriology (Morgan et al., 1983; Roberts et al., 1983). However this system had many drawbacks like radiation hazard, manual loading and unloading of vials, no inbuilt incubation system and lack of computer software. In low burden laboratories, fully automated systems like MB/Bact (Organon Teknika, Turnhout,
Belgium) and ESP II (Difco Laboratories, Detroit, Mich) serve the purpose but they have low capacity (Rohner et al., 1997). The fully automated, high capacity BACTEC MGIT 960 system is now being used as a rapid diagnostic system for tuberculosis in many developed countries. This system is easy to use, fully automated, non-invasive, nonradiometric with high performance. It has an oxygen sensitive fluorescent sensor embedded in silicon base which serves as an indicator of mycobacterial growth (Tortoli et al., 1999). Combination of a sold media with one broth-based method such as BACTEC 460 system is now widely accepted as "gold standard" (CDC, 1995). It is recommended that all the new diagnostic systems for tuberculosis must be evaluated fully (Chitra and Prasad, 2001). At present we are using BACTEC 460 as reference system along with conventional LJ media for culture of mycobacteria. The objective of this study is to evaluate the performance of BACTEC MGIT 960 system in terms of recovery rate, mean time to detection of mycobacteria and contamination rate and comparing it with reference BACTEC 460 system and LJ media. 


\section{MATERIALS AND METHODS}

The study was carried out at the department of microbiology, Armed Forces Institute of Pathology, Rawalpindi, Pakistan from May 2008 through Oct 2008. This laboratory receives various clinical samples from tertiary care hospitals like Combined Military Hospital, Military Hospital, Bone Marrow Transplant Centre and from Northern areas of Pakistan. A total of 260 consecutive clinical specimens (sputum 90, bronchoalveolar lavage 72 , pus 20 , tissue 36 , pleural fluid 12 , lymph node 10 , peritoneal fluid 10 , urine 6 , synovial fluid 3 and CSF 1) were dealt during the study period. For patients already on anti-tuberculous treatment, blood and bone marrow specimens and improper specimens like saliva and pus swabs were excluded from the study. Decontamination of specimens was carried out by standard N-acetyl-L-cysteine $\mathrm{NaOH}$ digestiondecontamination method (Kent and Kubica, 1985). A final concentration of $4 \% \mathrm{NaOH}$ was used for decontamination. A 2-3 drops of the processed specimens were placed on a glass slide to make smear, stained with Ziehl-Neelsen (ZN) acid fast staining and index is reported according to the protocol described by Ellis (Ellis and Zabrowarny, 1993). The remaining suspension was used for inoculation in both the liquid media (first in BACTEC 460) and then LJ medium.

\section{BACTEC 960 System}

Before inoculation of specimen into $7 \mathrm{~mL}$ MGIT tube, MGIT PANTA vial was reconstituted with $15 \mathrm{~mL}$ of MGIT growth supplement and mixed by gentle shaking. Each MGIT tube was checked for any turbidity or contamination and labelled properly. $0.8 \mathrm{~mL}$ of MGIT growth supplement/PANTA was added aseptically into MGIT tube with the help of insulin syringe. A $0.5 \mathrm{~mL}$ of the processed specimen was added to the tube and the cap was replaced immediately. The contents were mixed by inverting the tube 3-4 times. All the inoculated tubes were inserted in the MGIT 960 system after scanning the bar code. All the tubes were incubated at the temperature of $37{ }^{\circ} \mathrm{C}$. In case of a positive growth, the system automatically detects the growth and signals positive. After a maximum of six weeks (42 days), the instrument flags a tube negative if there is no growth. In case of a positive growth, the tube is taken out of the instrument, stained for AFB presence and subcultured on to Columbia agar (Oxoid, Basingstoke, UK) to look for any contamination. The day in which the tubes were kept inside the system for incubation was taken as day zero while the time to detection of mycobacteria was taken on the day of instrument positivity alone with AFB smear positivity. After confirmation of a positive growth in solid or broth based media, the parallel media for that particular specimen was read daily. When AFB were seen in a smear prepared from a positive MGIT tube, the growth was further confirmed as Mycobacterium tuberculosis complex (MTBC) by para-nitrobenzoic acid (PNB) test (Giampaglia et al., 2005). At the end of protocol incubation, all the instrument negative tubes were again subjected to ZN staining to look for false negative tubes.

\section{BACTEC 460 TB System}

All the $12 \mathrm{~B}$ vials containing $4 \mathrm{~mL}$ of modified Middlebrook $7 \mathrm{H} 12$ broth were supplemented with $0.1 \mathrm{~mL}$ of the antimicrobial supplement PANTA prior to inoculation of the specimens to minimize the chances of contamination. $0.5 \mathrm{~mL}$ of the specimen concentrate was inoculated in the BACTEC $46012 \mathrm{~B}$ vial and incubated aerobically at $37^{\circ} \mathrm{C}$ for up to 6 weeks. Growth of mycobacteria was monitored twice weekly in first two weeks and weekly thereafter for the next 4 weeks. A Growth index (GI) of 10 or more was considered tentatively positive and was read daily thereafter. An AFB smear was made when a GI of 100 or more was reached. Contamination of the medium was checked by Gram stain smears and by inoculation on to Columbia agar (Oxoid, Basingstoke, UK) containing 5\% horse blood (blood agar), incubated aerobically at $37^{\circ} \mathrm{C}$ overnight and examined for any growth. The positive growth showing presence of AFB was further confirmed as MTBC by radiometric $p$-nitro- $\alpha$-acetylamino- $\beta$-hydroxy propiophenone (NAP) test (Becton Dickinson Diagnostic Systems). At the end of protocol period, all the negative vials were stained with $\mathrm{ZN}$ stain to rule out false negative results.

\section{LJ Medium}

A $0.2 \mathrm{~mL}$ of the processed specimen was inoculated on two LJ slants that is one with PNB $(500 \mu \mathrm{g} / \mathrm{mL}$ of medium) and other without PNB (Giampaglia et al., 2005) and incubated at $37{ }^{\circ} \mathrm{C}$ in $5-10 \% \mathrm{CO}_{2}$ incubator. All the LJ slants were checked twice weekly for the first two weeks and then weekly for 8 weeks for the appearance of visible colonies on agar. Colonies of MTBC will not grow on LJ medium with PNB.

\section{Statistics}

Independent sample T-test was used to compare mean time to detection of mycobacteria and recovery rate in different culture systems/medium. $P$-value $<0.05$ was considered significant.

\section{Controls}

H37Ra ATCC 25177 of Mycobacterium tuberculosis was used as positive control. An uninoculated MGIT tube was used as negative control. Institutional control strain of Mycobacterium chelonei was used to check MGIT PNB and BACTEC NAP.

\section{RESULTS}

Out of total 260 specimens, 44 mycobacterial isolates (Mycobacterium tuberculosis complex, $n=43$; nontuberculous mycobacteria, $n=1$ ) were isolated from all the culture media. Pulmonary samples were among the 
dominant $(62.3 \%)$ followed by tissue $(13.8 \%)$ and pus (7.6\%). Maximum number of positive isolates 41 (93.2\%) were recovered from pulmonary specimens as shown in Figure 1, highlighting the heavy burden of pulmonary tuberculosis in our setup. Percentage of recovery of mycobacteria on BACTEC MGIT 960, BACTEC 460 and LJ medium was $97.6 \%, 93.0 \%$ and $83.7 \%$ respectively as shown in Table 1. P-value was less than 0.05 for recovery of mycobacteria between MGIT 960 and LJ medium which is a significant difference. No isolate was recovered from urine, pleural fluid, peritoneal fluid, synovial fluid and CSF. When combined together, BACTEC MGIT 960 and LJ medium detected 42 mycobacterial isolates (97.6\%) while BACTEC 460 plus LJ medium recovered 40 mycobacterial isolates (93.0\%). In combination with solid media, the recovery rates of both the liquid system were same as when considered individually. This is because of the fact that LJ medium gave positive growth only in smear positive specimens and all the smear positive specimens were also culture positive on both the liquid systems.

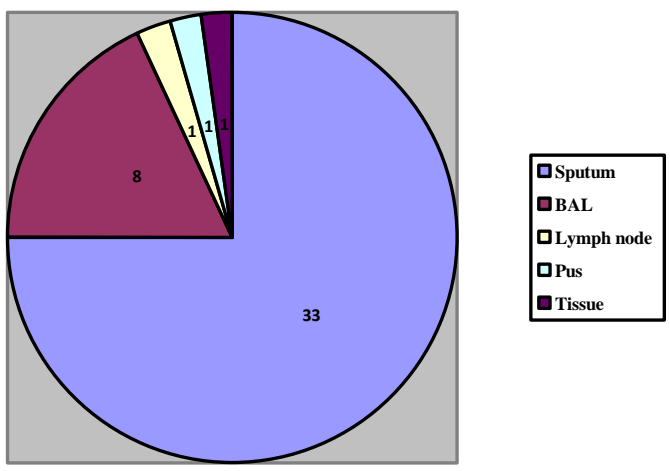

Figure 1: Number of mycobacterial isolates from various specimens $(n=44)$

Table 1: Relationship of concentrated specimen microscopy with mycobacterial culture on individual system/medium

\begin{tabular}{lllll}
\hline $\begin{array}{l}\text { Culture } \\
\text { system/media }\end{array}$ & $\begin{array}{l}\text { Smear positive } \\
\text { culture positive }\end{array}$ & $\begin{array}{l}\text { Smear negative } \\
\text { culture positive }\end{array}$ & $\begin{array}{l}\text { Smear negative } \\
\text { culture negative }\end{array}$ & Total \\
\hline MGIT 960 & 36 & 6 & 218 & 260 \\
BACTEC 460 & 36 & 4 & 220 & 260 \\
LJ medium & 36 & 0 & 224 & 260 \\
\hline
\end{tabular}

Mean time to detection of mycobacteria for smear positive specimens on BACTEC MGIT 960 system was 11.2 days and 14.2 days for smear negative specimens. It was 14.8 days on BACTEC 460 system for smear positive cases and 15.6 days for smear negative cases as shown in Table 2. Individual sample T-test was applied to check the significance level between mean time to detection of mycobacteria on both the liquid systems and $P$-value was found to be 0.037 which is a significant difference. Similarly a statistically significant difference was found between the individual liquid systems and LJ medium $(P<$ 0.05). Mean time to detection of mycobacteria was directly related to mycobacterial slide index as shown in Table 4.

Contamination rates on MGIT 960, BACTEC 460 and LJ medium were $9.6 \%, 5.6 \%$ and $3.4 \%$ respectively. Contamination rates were high in the initial two months but then decreased gradually in the subsequent months as shown in Table 3.
Table 2: Relationship of mean detection time of mycobacteria with smear positivity on individual systems

\begin{tabular}{lll}
\hline \multirow{2}{*}{$\begin{array}{l}\text { BACTEC } \\
\text { system }\end{array}$} & $\begin{array}{l}\text { Mean detection time in days of } \\
\text { mycobacteria (range) }\end{array}$ \\
\cline { 2 - 3 } & AFB $(+)$ smear & AFB $(-)$ smear \\
\hline MGIT 960 & $11.2(1-30)$ & $14.2(6-30)$ \\
BACTEC 460 & $14.8(2-31)$ & $15.6(3-36)$ \\
LJ medium & $28.3(16-52)$ & - \\
\hline
\end{tabular}

Table 3: Contamination rates (\%) on individual system

\begin{tabular}{lllll}
\hline $\begin{array}{c}\text { Culture } \\
\text { System }\end{array}$ & $\begin{array}{c}\text { May- } \\
\text { June }\end{array}$ & $\begin{array}{c}\text { July- } \\
\text { Aug }\end{array}$ & $\begin{array}{c}\text { Sept- } \\
\text { Oct }\end{array}$ & $\begin{array}{c}6 \text { Months } \\
\text { Average }\end{array}$ \\
\hline MGIT 960 & 14.5 & 9.8 & 4.7 & 9.6 \\
BACTEC 460 & 7.8 & 4.1 & 5.1 & 5.6 \\
LJ medium & 3.8 & 3.4 & 2.9 & 3.4 \\
\hline
\end{tabular}


Table 4: Relationship of mycobacterial index with detection time of mycobacteria on individual system $/ \mathrm{medium}$

\begin{tabular}{lllll}
\hline $\begin{array}{l}\text { Mycobacterial } \\
\text { Index }\end{array}$ & $\begin{array}{l}\text { Number of } \\
\text { mycobacterial } \\
\text { isolates }\end{array}$ & \multicolumn{3}{l}{ Mean detection time in days } \\
\cline { 2 - 5 } & 7 & MGIT 960 & BACTEC 460 & LJ \\
\hline $4+$ & 12 & $8.2(1-15)$ & $9.7(3-16)$ & $20.2(16-22)$ \\
$3+$ & 12 & $9.9(3-20)$ & $14(3-26)$ & $24.8(18-28)$ \\
$2+$ & 5 & $11.8(3-22)$ & $16.3(5-30)$ & $29.0(25-38)$ \\
$1+$ & & $12.4(5-25)$ & $16.8(6-30)$ & $39.1(34-52)$ \\
\hline
\end{tabular}

\section{DISCUSSION}

Due to increase in the spread of tuberculosis worldwide, early diagnosis and prompt treatment has become important for the control of this disease (ATS, 2000). The advancement in development of new diagnostic methods is an important element of the "Global Plan to Stop TB" and the WHO new global "Stop TB Strategy" (Pai et al., 2008). According to WHO study in 2006 , it has been found that the world's expenditure per year on diagnosis of TB is about US $\$ 1$ billion (WHO, 2006). The methods presently used for the diagnosis and sensitivity of mycobacteria are either costly or time consuming. Therefore, a rapid, cost effective and simple method is required for under developed countries. Although BACTEC 460 system is being withdrawn worldwide, its use in recent years have significantly improved the recovery rate of mycobacteria but the culture procedure on this system is time consuming and labor-intensive. MGIT 960 system has been developed recently for the rapid diagnosis and sensitivity testing of mycobacteria (Somoskovi et al., 2004). It has also overcome the drawbacks of previous BACTEC 460 system which was and still being used as a gold standard for the culture of mycobacteria in various countries (Ganeswire et al., 2004).

A number of studies have been conducted worldwide to evaluate the diagnostic efficacy of MGIT 960 system from various clinical specimens. We evaluated the performance of the new MGIT 960 system in terms of recovery rate of $\mathrm{MTBC}$, mean time to detection of mycobacteria and contamination rates from various clinical specimens in our settings and compared it with the already in use gold standard BACTEC 460 system and conventional LJ medium. In our study, out of total 260 clinical specimens, 44(16.9\%) yielded growth of mycobacteria. $15.9 \%$ were MTBC and only one isolate was nontuberculous mycobacteria (NTM). Four isolates of MTBC were recovered only on MGIT 960 system and not on BACTEC 460 system while only one isolate was recovered on BACTEC 460 system but not on MGIT 960 system and that was identified as NTM. The possible reason for recovery of four isolates only on MGIT 960 and not on BACTEC 460 could be that the mycobacteria present in the $12 \mathrm{~B}$ vial did not metabolize the $\mathrm{C}^{14}$ palmitic acid completely. Another reason could be that the MGIT 960 tube contains $7 \mathrm{~mL}$ broth while $12 \mathrm{~B}$ vial contains $4 \mathrm{~mL}$ broth. The high volume of broth in MGIT 960 tube might have diluted the potential growth inhibitors thus providing a better chance for mycobacteria to grow. A number of other studies have revealed that all the isolates of MTBC or NTM were not recovered on both the systems (Somoskovi et al., 2004). The possible reason why the only NTM in our study was not recovered on MGIT 960 system might be that concentrated specimen was first inoculated into BACTEC 460 vial followed by MGIT 960 tube. The number of organisms might be low in the sample and uneven distribution had resulted in negative culture in MGIT 960 system.

Our study showed that mean time to detection of mycobacteria on MGIT 960 was shorter than BACTEC 460 and LJ medium. The minimum time to detection was one day on MGIT 960 and 2 days on BACTEC 460. Maximum time to detection was 30 days on MGIT 960 and 36 days on BACTEC 460 . Findings of our study were also consistent with another study (Hanna et al., 1999). They found that the mean time to detection of mycobacteria was 14.4 days for MGIT 960 and 15.2 days for BACTEC 460. The detection time of mycobacteria has a vital role in the performance of a system. Early detection of mycobacteria will limit the spread of infection by timely treatment.

During the course of study, it was observed that mycobacterial growth is seen as small clumps, grains or cords settling down at the bottom of tube after shaking like snow fall when visualized in good light while contamination appeared as uniform turbidity in the entire tube. Contamination rate was higher on MGIT 960 system $(9.6 \%)$ as compared to BACTEC 460 system (5.6\%). Higher contamination rate is the major draw back of the fully automated system. However in a study (Somoskovi et al., 2004), they found that the contamination rates on MGIT 960 and BACTEC 460 were $3.7 \%$ and $2.9 \%$ respectively. In another study (Rishi et al., 2007), contamination rate on MGIT 960 was $13.4 \%$ and $27.2 \%$ on LJ medium which is very high. The higher contamination rate on MGIT 960 may be due to the fact that the cap of MGIT 960 tube is opened for specimen inoculation while insulin syringes are used in BACTEC 460 vial for inoculation without opening them. Another reason could be that MGIT 960 system uses enriched broth whereas the 12B broth medium in BACTEC 460 system is low in nutrients as it uses $C^{14}$ palmitic acid. Thus the enriched high volume broth is like a double-edge sword, at one side it dilutes the inhibitors and improves the recovery of mycobacteria while on the other side, enrichment favors the contaminants.

Another interesting observation was that the contamination rate for MGIT 960 system was high in the 
first two months of the study but it declined significantly in next four months. This could be due to the fact that it was a new system introduced in our laboratory, and laboratory staff were not well familiar with the system initially but their handling of the system improved significantly in subsequent months once they gained experience. Another possibility could be that climatic conditions in our country are hot in the month of May, June and July and transportation was also delayed in some specimens thus allowing the contaminants to overgrow.

In our study we observed that maximum number $(77.5 \%)$ of mycobacterial isolates were cultured from sputum samples indicating that sputum is the most frequent specimen submitted by the patients. This also signifies the importance of early isolation of these cases and prevention of spread of bacilli from open or infectious cases.

There were no false negative results in our study that is all the MGIT 960 instrument- negative tubes (at the end of 42-day protocol) were examined by direct AFB microscopy and subcultured to solid media. We observed three false positive result in our study that is instrument positive but smear negative and subculture negative but after incubation for 3 to 4 more days, AFB was detected under direct microscopy.

The non-radiometric, fully automated, $7 \mathrm{~mL}$ BACTEC MGIT 960 system is rapid in terms of recovery of mycobacteria and time to detection of mycobacteria as compared with radiometric, semi-automated BACTEC 460 system and conventional LJ medium. It has eliminated the use of needles for inoculation or testing of vials. It has high capacity and less labor-intensive with minimum staff required. As this system uses plastic tubes so there are less chances of breakage and laboratory accidents. The average cost per test that is AFB culture/sensitivity on LJ medium is $4.7 \$$ (400 PKRs) and 9.4\$ (800 PKRs) on BACTEC MGIT 960 system. Although expensive, but BACTEC MGIT 960 system can be used as a reliable alternative for culture of mycobacteria in high burden laboratories keeping in view the financial resources of the laboratory.

\section{REFERENCES}

American Thoracic Society (ATS). (2000). Diagnostic standards and classification of tuberculosis in adults and children. American Journal of Respiratory and Critical Care Medicine 161, 1376-1395.

Centers for Diseases Control. (1995). Essential components of a tuberculosis prevention and control program. Recommendations of the Advisory Council for the elimination of tuberculosis. Morb Mortal 44, 1 16.

Chitra, C. and Prasad, C. E. (2001). Evaluation of MGIT for primary isolation of Mycobacteria from clinical samples. Indian Journal of Tuberculosis 48, 155-156.

Ellis, R. C. and Zabrowarny, L. A. (1993). Acid fast bacilli staining protocol. Journal of Clinical Pathology 46, 559-560.
Farnia, P., Mohammadi, F., Zarifi, Z., Tabatabee, D. J., Ganavi, J., Ghazisaeedi, K., Farnia, P. K., Gheydi, M., Bahadori, M., Masjedi, M. R. and Velayati, A. A. (2002). Improving sensitivity of direct microscopy for detection of acid-fast bacilli in sputum: Use of chitin in mucus digestion. Journal of Clinical Microbiology 40, 508-511.

Ganeswire, R., Chui, C. S., Balan S. and Puthucheary S.D (2004). Comparison of BACTEC MGIT 960 system and BACTEC 460 TB system for growth and detection of Mycobacteria from clinical specimens. Malaysian Journal of Pathology 26(2), 99-103.

Giampaglia, C. M. S., Martins, M. C., Inumaru, V. T. G., Butuem, I. V. and Telles, M. A. S. (2005). Evaluation of a rapid differentiation test for the Mycobacterium tuberculosis complex by selective inhibition with $p$ nitrobenzoic acid and thiophene-2-carboxylic acid hydrazide. International Journal of Tuberculosis and Lung Disease 9(2), 206-209.

Hanna, B. A., Ebrahimzadeh, A., Elliott, L. B., Morgan, M. A., Novak, S. M., Rusch-Gerdes, S., Acio, M., Dunbar, D. F., Holmes, T. M., Rexer, C. H., Savthyakumar, C. and Vannier, A. M. (1999). Multicenter evaluation of the BACTEC MGIT 960 system for recovery of mycobacteria. Journal of Clinical Microbiology 37, 748-752.

Hasan, Z., Tanveer, M., Kanji, A., Hasan, Q., Ghebremichael, S. and Hasan, R. (2006). Spoligotyping of Mycobacterium tuberculosis isolates from Pakistan reveals predominance of central Asian strain 1 and Beijing isolates. Journal of Clinical Microbiology 44, 1763-1768.

Kent, P. T. and Kubica, G. P. (1985). Public health mycobacteriology, a guide for the level III laboratory. Centers for Disease Control. Development of Health and Human Services, Atlanta, Ga.

Morgan, M. A., Horstmeier, C. D., DeYoung, D. R. and Roberts, G. D. (1983). Comparison of a radiometric method (BACTEC) and conventional culture media for recovery of mycobacteria from smear negative specimens. Journal of Clinical Microbiology 18, 384388.

Pai, M., Ramsay, A. and O'Brien, R. (2008). Evidencebased tuberculosis diagnosis. PLoS Medicine 22 5(7), 156.

Rishi, S., Sinha, P., Malhotra, B. and Pal, N. (2007). A comparative study for the detection of mycobacteria by BACTEC MGIT 960, Lowenstein Jensen media and direct AFB smear examination. Indian Journal of Microbiology 25, 383-386.

Roberts, G. D., Goodman, N. L., Heifets, L., Larh, H. W., Lindner, T. H., McClatchy, J. K., McGinnis, M. R., Siddiqi, S. H. and Wright, P. (1983). Evaluation of the BACTEC radiometric method for recovery of mycobacteria and drug susceptibility testing of Mycobacterium tuberculosis from acid-fast smearpositive specimens. Journal of Clinical Microbiology 18, 689-696.

Rohner, P. B., Ninet, C., Metral, S., Emler and Auckenthaler, R. (1997). Evaluation of the MB/BacT 
system and comparison to the BACTEC 460 system and solid media for isolation of mycobacteria from clinical specimens. Journal of Clinical Microbiology 35, 3127-3131.

Somoskovi, A., Kodmon, C., Lentos, A., Bartfai, Z., Tamasi, L., Fuzy, J. and Magyar, P. (2004). Comparison of recoveries of Mycobacterium tuberculosis using BACTEC MGIT 960, the BACTEC 460 TB System and Lowenstein-Jensen Medium. Journal of Clinical Microbiology 38, 2395-2397.

Tortoli, E., Cichero, P., Piersimoni, C., Simonetti, M. T., Gesu, G. and Nista, D. (1999). Use of BACTEC MGIT 960 for recovery of mycobacteria from clinical specimens: multicenter study. Journal of Clinical Microbiology 37, 3578-3582.

World Health Organization (WHO). (2006). Special programme for research and training in tropical diseases. Diagnostics for tuberculosis. Global demand and market potential. 